\title{
Truncal valve repair and other aortic pediatric valves
}

\author{
Ismail Bouhout, Nancy Poirier \\ Department of Cardiac Surgery, CHU Sainte Justine, University of Montreal, Montreal, Canada \\ Correspondence to: Nancy Poirier, MD, Division of Cardiac Surgery, CHU-ME Ste-Justine, University of Montreal, 3175 Côte Ste-Catherine, \\ Montreal QC H3T 1C, Canada. Email: ncpoirier@videotron.ca.
}

Submitted Apr 08, 2019. Accepted for publication Apr 13, 2019.

doi: 10.21037/acs.2019.05.10

View this article at: http://dx.doi.org/10.21037/acs.2019.05.10

\section{Clinical vignette}

A 13-year-old male is diagnosed at birth with a type 1 truncus arteriosus. He underwent correction at three days of life without intervention on the truncal valve (TV) with a $12 \mathrm{~mm}$ pulmonary homograft. He now presents with severe symptomatic TV regurgitation.

\section{Surgical techniques}

\section{Preparation}

Preoperative trans-esophageal echocardiography (TEE) is performed in all patients to assess the reparability of the valve. On the short-axis TEE, the morphology of the valve is assessed by determining the number of leaflets, commissures and raphes. Specific focus is placed on assessing cusp mobility, the presence of calcification, and symmetry of commissures/raphes. In the long-axis view, the severity and the mechanism of the regurgitation is determined as suggested by the direction of the regurgitant jet. In the present case, the truncal valve is quadricuspid with three well-developed cusps and one marginally smaller cusp. The regurgitant jet is centrally directed. The leaflets are mobile with no calcification. In most cases, the quadricuspid TV regurgitation is due to cusp restriction, making reparability of the valve more challenging, depending on the relative dimensions of the non-retracted cusps.

\section{Exposition and preparation}

The procedure is performed through a full median sternotomy. The cardiopulmonary bypass is initiated with the proximal arch and bicaval cannulation. Complete aortotomy is performed one $\mathrm{cm}$ above the sino-tubular junction to expose the aortic valve (AV). Cardioplegia is initiated antegrade and intermittently administered directly through the coronary ostia using Del Nido solution. Traction stitches are placed on each commissure for exposition. The valve is evaluated to determine the thickening, pliability, mobility and the degree of retraction of each cusp. Each cusp length is then measured using a silk free tie. Polypropylene sutures are then placed at the tip of the coapting cusps to visually assess valve geometry prior to repair. A Hegar dilator chosen according to the patient's body surface area is passed to assess any left ventricular outflow tract (LVOT) obstruction. Finally, the effective height of each is measured using a dedicated caliper to determine any prolapse.

\section{Operation}

A number of techniques have been previously described to repair a variety of AVs. A combination of these procedures must be used in TV repair. They address cusp prolapse (triangular resection of cusp free edge, cusp plication and commissural resuspension), annular dilatation (circumferential suture annuloplasty and subcommissural plication) and cusp shaving for valve dysplasia or thickening. Nevertheless, AV repairs at large in congenital patients can be more complex procedures, especially in patients with previous balloon valvuloplasty and varying degrees of cusp retraction and thickening. These patients may require cusp extensions with autologous or bovine pericardium.

Our patient had four cusps of approximately equal size, thus allowing successful bicuspidization. The cusps and commissure/raphe were aligned such that a bicuspid valve with commissures at $180^{\circ}$ configuration in an antero- 
posterior axis could be created. Two facing commissures were extensively mobilized to maximize leaflet mobility, excursion and geometric height. Interrupted 5-0 polypropylene sutures were then placed between the rightleft coronary cusps and between the posterior and anterior non-coronary cusps creating the bicuspid configuration.

In the case of unequal leaflets, a tricuspidization technique is preferred. The rudimentary cusp is removed along with the corresponding sinus. The truncal valve wall is then reapproximated which reduce the aortic annulus. In the presence of two small cusps, a tricuspidization is achieved by unifying the two rudimentary leaflets as described for bicuspidization.

\section{Completion}

The final step to any AV repair is to evaluate the repair result and predict durability. First, the valve opening is assessed by passing a Hegar dilator chosen according to the patient's body surface area. This step is important as moderate aortic stenosis following AV repair is reported in up to $10 \%$ of pediatric patients (1). Secondly, a dedicated caliper is used to measure the final cusp effective height. While the effective height as a measure of long-term durability is well studied in adults (2), the target measure in pediatric patients remains unknown. Finally, the dynamic result is evaluated using TEE with a special focus on the residual $\mathrm{AV}$ regurgitation, the direction of the jet, the mean aortic gradient and the echocardiographic effective height.

\section{Comments}

AV repair is an attractive option in pediatric patients. It avoids the long-term risks of anticoagulation, allows growth and postpones AVR to an older age where a more definite AV substitute could be implanted. In the last decade, there has been a threefold increase in $\mathrm{AV}$ repairs (1) partly because of favourable results published in adults with low reintervention rates and long-term valverelated complications $(1,3)$. In recent published cohorts, the freedom from $\mathrm{AV}$ reintervention following pediatric valve repairs ranged between $50 \%$ and $80 \%$ at 10 years, with a five-year median time to reoperation (1). Various factors are predictive of reintervention including preoperative aortic stenosis, unicuspid AVs, lack of coaptation symmetry and the use of patch extension. Truncal valves are a subgroup of patients where AV repair is challenging. The Melbourne group has recently published a freedom from valve reoperation of $34 \%$ at ten years in patients with a previous TV repair (4). Interestingly, in a study examining patients who underwent TV repair, leaving a quadricuspid valve after repair was associated with a trend towards a higher risk of reoperation (5). Therefore, when feasible, a bicuspidization or tricuspidization repair technique should be attempted in order to improve long-term outcomes. None of these repair techniques have been demonstrated to be superior in terms of durability and surgical technique should be tailored according to the patient's AV disease.

\section{Acknowledgments}

None.

\section{Footnote}

Conflict of interest: The authors have no conflicts of interest to declare.

\section{References}

1. Bouhout I, Ba PS, El-Hamamsy I, et al. Aortic Valve Interventions in Pediatric Patients. Semin Thorac Cardiovasc Surg 2019;31:277-87.

2. Aicher D, Kunihara T, Abou Issa O, et al. Valve configuration determines long-term results after repair of the bicuspid aortic valve. Circulation 2011;123:178-85.

3. Aicher D, Fries R, Rodionycheva S, et al. Aortic valve repair leads to a low incidence of valve-related complications. Eur J Cardiothorac Surg 2010;37:127-32.

4. Naimo PS, Fricke TA, d'Udekem Y, et al. Impact of truncal valve surgery on the outcomes of the truncus arteriosus repair. Eur J Cardiothorac Surg 2018;54:524-31.

5. Myers PO, Bautista-Hernandez V, del Nido PJ, et al. Surgical repair of truncal valve regurgitation. Eur J Cardiothorac Surg 2013;44:813-20.
Cite this article as: Bouhout I, Poirier N. Truncal valve repair and other aortic pediatric valves. Ann Cardiothorac Surg 2019;8(3):436-437. doi: 10.21037/acs.2019.05.10 